\title{
LIXUS Y MORDELLISTENA LARVAS DE COLEÓPTEROS BARRENADORES DE TALLO EN PLANTAS DE RAMA NEGRA (CONYZA BONARIENSIS L.)
}

\author{
JuAn, V. F. ${ }^{1}$; Martinoia, G.I. ${ }^{2}$; Saint ANDRÉ, H.M. ${ }^{1}$ \& NúÑEZ Fré, F.R. ${ }^{1,3}$
}

\begin{abstract}
RESUMEN
En el centro de la Provincia de Buenos Aires, durante la campaña agrícola 2014/2015 se registraron dos larvas endofíticas barrenadoras del tallo en rama negra. Para su identificación y estudio, ejemplares recolectados en las malezas fueron llevados al laboratorio para su disección. Las larvas encontradas correspondieron a dos insectos del Orden Coleóptera de las familias Curculionidae y Mordellidae. El barrenador Curculionidae pertenece al género Lixus y el de la familia Mordellidae es del género Mordellistena. Los adultos fueron obtenidos sobre las plantas trasplantadas y en los muestreos a campo sobre ejemplares de rama negra y en algunos casos refugiados dentro de las galerías. Si bien estos organismos pueden comportarse como enemigos naturales de rama negra, hay antecedentes de larvas de insectos barrenadores que interfieren con las prácticas de control químico, por limitar el transporte de herbicidas sistémicos.

Palabras claves: Conyza spp.; Coleoptera; Larvas barrenadoras; Insectos endofíticos.

Lixus and Mordellistena stem borer larvae in Hairy Fleabane (Conyza bonaeriensis L.) plants
\end{abstract}

\section{ABSTRACT}

\section{Lixus and Mordellistena stem borer larvae in Hairy Fleabane (Conyza bonaeriensis L.) plants}

In the center of Buenos Aires Province, during the crop year 2014/2015 two endophytic stem borer larvae were found in Hairy Fleabane plants. In order to study and identify the species, samples of this weed were collected and taken to the laboratory for dissection. The larvae corresponded to two insects of the Coleoptera order, Curculionidae and Mordellidae families. The Curculionidae borer belongs to the genus Lixus and the Mordellidae borer was identified as Mordellistena genus. Adults

1.- Catedra de Terapéutica Vegetal. Facultad de Agronomía, Universidad Nacional del Centro de la Provincia de Buenos Aires, Av. República de Italia 780. (7300) Azul, provincia de Buenos Aires. Teléfono/Fax: 02281 433291/92/93. Email vjuan@faa.unicen.edu.ar

2.- Cátedra de Zoología Agrícola. Facultad de Agronomía (UNICEN).

3.- Becario Perfeccionamiento Comisión de Investigaciones Científicas de la Provincia de Buenos Aires, CIC-PBA.

Manuscrito recibido el 27 de julio de 2015 y aceptado para su publicación el 13 de noviembre de 2015. 
V. F. Juan et al.

individuals were obtained from Hairy Fleabane in pots, and from field sampling plants, in some cases within the galleries. Even if these individuals can act as natural enemies of Hairy Fleabane, it must be pointed out that some background information mentions larvae of stem-boring insects, that may cause a negative effect on chemical control practices, as they limit the transport of systemic herbicides.

Key words: Conyza spp.; Coleoptera; Stem borer larvae; Endophagus.

\section{INTRODUCCIÓN}

El género Conyza pertenece a la familia de las Asteraceae, e incluye aproximadamente 50 especies, que se distribuyen en casi todo el mundo (Kissmann y Groth, 1999). A nivel mundial, las principales malezas de este género son Conyza canadensis (L.) y Conyza bonariensis. La primera es originaria de América del Norte, mientras que $C$. bonariensis es nativa de América del Sur y está presente en forma abundante en Argentina, Brasil, Paraguay y Uruguay, aunque también se ha registrado en Colombia y Venezuela infestando cultivos de café (Kissmann y Groth, 1999).

En nuestro país esta especie es denominada comúnmente "rama negra” y en el área sojera existen plantas con marcadas diferencias morfológicas, que de acuerdo a identificaciones taxonómicas han sido consideradas como dos especies: Conyza bonariensis y Conyza sumatrensis, aunque algunos botánicos afirman que se trata de distintas variedades de Coniza bonariensis (Nisensohn et al., 2011).

En la Argentina, antes de la década del 80, esta maleza era sólo problemática en pasturas, dado que en las áreas con cultivos intensivos y extensivos se utilizaba la labranza convencional, un sistema en el cual esta especie no parece prosperar (Brown y Whitwell, 1988).
En los últimos años, la misma se ha presentado como invasora en los sistemas en siembra directa de la región pampeana y actualmente se la considera una especie de difícil control con la tecnología disponible. Las plantas en estado adulto son tolerantes a los tratamientos con glifosato a las dosis normales de uso (Faccini et al., 2008).

Si bien hay abundante información sobre estrategias de manejo con herbicidas, no se ha considerado la influencia de insectos que afectan a esta especie y el posterior resultado de estos tratamientos químicos.

A nivel mundial, existen algunos reportes de control deficiente con glifosato en diversas malezas, en las cuales se ha observado la presencia de insectos realizando galerías en el tejido vascular de las plantas: Agropyron repens (Westra et al., 1981), Ambrosia trifida (Ott et al., 2005) у Chenopodium album (Harder et al., 2007).

En la zona centro de la Provincia de Buenos Aires, se ha detectado la presencia de dos larvas endófagas barrenadoras del tallo, que provocan galerías en plantas de rama negra.

El objetivo del presente estudio fue relevar la presencia e identificar los agentes causales del daño observado sobre ejemplares de Conyza bonariensis que crecen como malezas en lotes agrícolas de la zona centro de la Provincia de Buenos Aires. 